Federal Reserve Bank of Minneapolis

Research Department

\title{
Prizes and Patents: Using Market Signals to Provide Incentives for Innovations
}

\author{
V. V. Chari, Mikhail Golosov, and Aleh Tsyvinski*
}

Working Paper 673

August 2009

\begin{abstract}
Innovative activities have public good characteristics in the sense that the cost of producing the innovation is high compared to the cost of producing subsequent units. Moreover, knowledge of how to produce subsequent units is widely known once the innovation has occurred and is, therefore, non-rivalrous. The main question of this paper is whether mechanisms can be found which exploit market information to provide appropriate incentives for innovation. The ability of the mechanism designer to exploit such information depends crucially on the ability of the innovator to manipulate market signals. We show that if the innovator cannot manipulate market signals, then the efficient levels of innovation can be implemented without deadweight losses - for example, by using appropriately designed prizes. If the innovator can use bribes, buybacks, or other ways of manipulating market signals, patents are necessary.
\end{abstract}

\footnotetext{
${ }^{*}$ Chari: University of Minnesota and Federal Reserve Bank of Minneapolis; Golosov: MIT and New Economic School; Tsyvinski: Yale and New Economic School. The authors thank the National Science Foundation for support. The views expressed herein are those of the authors and not necessarily those of the Federal Reserve Bank of Minneapolis or the Federal Reserve System.
} 


\section{Introduction}

Prosperity and economic growth depend fundamentally on innovation, that is, on the production of new ideas, goods, techniques, and processes. A widely shared belief is that competitive markets, on their own, will produce an inadequate supply of innovation. One argument that supports this belief is that many types of innovation have public good characteristics. The cost of producing an idea or the first unit of a good is large. The cost of replicating an idea or producing copies of an innovation is small, especially compared to the cost of innovating. In the absence of intellectual property rights, competitive markets will produce duplicates and sell them at essentially marginal cost. The producer of the first unit of the good will then be unable to recoup the costs of innovation and will rationally choose not to innovate.

An extensive literature on innovation has discussed the efficiency of various mechanisms intended to increase the level of innovation above that produced by the competitive markets. ${ }^{1}$ The central question in the theory of intellectual property rights is to determine the best mechanism that weighs the social benefits of innovation against the costs of distortions imposed by the mechanism. One frequently used mechanism is the patent system, which grants property rights to innovators for some period of time and prevents competitors from copying the innovation. Granting monopoly rights of this form induces innovation by allowing inventors to recoup the costs of an innovation. However, patents impose the usual deadweight costs of monopoly on the society. The classic analysis of patents (see, e.g., Nordhaus 1969) weighs the costs of monopoly distortions against the benefits of encouraging innovation.

An alternative mechanism is to award prizes. ${ }^{2}$ Prizes reward innovators while making the fruits of the innovation public. Competitive markets then produce an efficient number of units of the good or exploit the idea associated with the innovation as efficiently as possibly. This mechanism has the advantage that it avoids the monopoly distortions associated with patents. The disadvantage of this mechanism is that it requires the entity awarding prizes to have a great deal of information about the social value of the innovation. This social value is often not directly available to the prize giver. Thus, an important question is, how can the prize giver use information from competitors in the industry or, more generally, from the market to elicit the social value of the innovation?

This question is particularly interesting in the context of the theory of innovation because

\footnotetext{
${ }^{1}$ See Scotchmer (2004) for a comprehensive treatment.

${ }^{2}$ The classic analysis by Wright (1983) discusses patents and research prizes. See also Hopenhayn, Llobet, and Mitchell (2006) for a modern mechanism design treatment of prizes, patents, and buyouts.
} 
those who argue that innovation has public good characteristics explicitly assume that copies of innovated goods can be produced at little more than production costs. In other words, once the good is invented, competitors in the marketplace have a great deal of information on how to produce the good in question. The social value of the good depends crucially on the number of units of the good that will be sold in the competitive marketplace. Any theory of patents as a form of intellectual property must ask why mechanisms cannot be devised which exploit information that will become available in the marketplace after the good has been innovated.

In this paper, we ask whether market signals can be used to reward innovation appropriately while avoiding the deadweight costs of monopoly. We answer this question by setting up a general mechanism design framework. In this framework, a planner can use information from innovators, competitors, and the marketplace to reward the innovator. We first consider an environment in which the innovator cannot manipulate the information about the value of the innovation. We show that a prize-like mechanism can induce socially efficient levels of innovation and completely avoid the deadweight losses of monopoly. We then show how to construct a mechanism that yields a socially efficient outcome as a unique equilibrium. In terms of implementation, such mechanisms may take a variety of forms. For example, we show that a mechanism that makes the prize for the innovation a function of total sales in competitive markets can implement socially efficient levels of innovation.

We then analyze several classes of environments where the innovator can manipulate market signals. Two forms of market manipulation are of particular interest: bribes and buybacks. In terms of bribes, we assume that the innovator can make binding commitments to make payments to market participants. For example, if the mechanism involves the use of prizes which are functions of aggregate sales, we allow innovators to bribe other producers to induce them to misrepresent sales. We show that bribes of this kind can be used by the innovator to subvert market signals completely. Indeed, we show that the best mechanism necessarily resembles patents. Society must then necessarily incur the deadweight costs of monopoly to induce innovations. One qualification to this result is that the associate bribes may need to be large. Indeed, if bribes are restricted to be bounded, but prizes can be larger than the maximal bribes, socially efficient levels of innovation can be implemented by prize-like mechanisms.

We examine the possibility that implicit collusion through repeated interaction is a bribe-like mechanism. We show that implicit collusion may indeed substitute for bribes but that the size of the implied bribes is necessarily bounded. If prizes can be larger than this bound, implicit collusion 
through repeated interaction is not, by itself, a barrier to the use of prizes to ensure efficiency.

In terms of other forms of market manipulation, we show that prize-like mechanisms are vulnerable to hidden buybacks. That is, prizes can be manipulated by the innovator secretly purchasing the good so as to make it seem that the market size is larger than it is. We show that, if the costs of these buybacks are small relative to the costs of the innovation, any mechanism that induces innovation must necessarily induce patents.

The main contribution of this paper is to show that the desirability of the patents as a mechanism to induce innovation relies crucially on the ability of the innovator to manipulate signals. If such manipulation is relatively easy, patents are necessary. If manipulation is costly, patents are harmful.

We use our general mechanism design approach to analyze a variety of specific proposals to replace patents by alternative mechanisms intended to spur innovation while avoiding the deadweight costs of monopoly. Kremer (1998) has forcefully argued that a particular mechanism, patent buyouts, can induce an efficient level of innovation without the costs of monopoly. His mechanism utilizes an auction to buy patents. Such an auction exploits information that competitors and, more generally, the market have in determining the terms of the buyout.

We show that Kremer's auction is susceptible to manipulation by the innovator. Specifically, suppose that the innovator can use one or more accomplices to participate in the auction. We show that Kremer's auction leads to inefficient outcomes. If the auction designer can exclude the innovator or his accomplices from participating in the auction, the mechanism does indeed yield efficient outcomes. Thus, the desirability of Kremer's mechanism relies on the ability to preclude the manipulation of the mechanism by the innovator. Recently, the Advanced Market Commitments (AMC) plan has been set up with the active participation of a number of governments and nongovernmental organizations. This plan proposes to subsidize, at deeply discounted prices, the vaccine manufacturers who sell vaccines which protect against tropical diseases to developing countries. The mechanism makes the amount of the subsidy a function of the number of doses of vaccine sold by the pharmaceutical company. The mechanism is intended to allow vaccine manufacturers to recoup the cost of innovation while ensuring that vaccines are sold at the marginal cost of production. Our main result shows that this mechanism is vulnerable to hidden buybacks. Vaccine manufacturers have strong incentives to buy, or have accomplices buy, dosages of the vaccine secretly. If such buybacks are easy to implement, the mechanism used by the AMC plan is likely to yield highly inefficient outcomes. If hidden buybacks are privately costly, this mechanism is likely to do well in 
stimulating innovations while avoiding deadweight losses.

In terms of applications and designing mechanisms in practice, the main message of our paper is that we should be cautious about adopting proposed new mechanisms. Such mechanisms require consideration of how to make them manipulation proof. We have raised concerns about manipulation of three specific kinds. For mechanisms that condition rewards on the quantity of the units sold on the market, market manipulation can be conducted through buybacks as well as attempts by innovators to set prices artificially low to stimulate demand. The second mechanism is explicit or implicit bribes. The third type of market manipulation that is particularly relevant in the context of auctions is the use of accomplices to submit fake bids.

There is a small literature on how information available on the market can be used in designing rewards for innovation. ${ }^{3}$ Kremer (1998) is the most influential recent paper with a detailed prize reward mechanism. As we have argued above, his mechanism is subject to the possibility of manipulation. Guell and Fischbaum (1995) propose a mechanism which uses sales on a test market for a relatively short period of time to obtain an estimate of the social surplus. Once such information is received, the government extrapolates this information to obtain an estimate of the total value of the social surplus if a good were to be sold on the total market. Then the innovator receives a prize with the value equal to the estimated surplus. This proposal is certainly subject to market manipulation. The innovator has strong incentives to increase the demand in the test market. In the most plausible cases, if one assumes that the marginal cost of production is small compared to the value of the innovation, and if one assumes that the monopolist can sell the good at zero price, then this mechanism leads to extremely inefficient outcomes. Shavell and van Ypersele (2001) propose an optional reward system in which they allow an innovator to either stay with the patent or choose a buyout reward. Their mechanism has rewards only if the lowest social payoff is positive. If such an assumption does not hold, patents are optimal.

\section{Model}

Consider an economy in which an innovator has an idea of quality $\theta$. This idea can be transformed into a good of quality $\theta$ if a fixed cost of $K>0$ is incurred. If this cost is not incurred, a good of quality $\theta=0$ is produced. We assume that the quality of the innovation $\theta \in[0, \bar{\theta}]$ and is distributed according to the cumulative distribution function $F(\theta)$. The social value of the innovation under competitive markets is given by $S(\theta)$, where $S^{\prime}(\cdot)>0, S(0)=0$.

\footnotetext{
${ }^{3}$ See Abramowicz (2003) for a review of a variety of proposals.
} 
We normalize profits if a good is produced under the competitive markets to be equal to zero. The good can also be produced by a monopoly. Let the monopoly profits be given by $\pi(\theta)$, where $\pi^{\prime}(\cdot)>0, \pi(0)=0$. We assume that monopoly conveys deadweight costs. The social value of the innovation under monopoly, $S^{m}(\theta)$, is smaller than the social value of the innovation under competitive markets:

$$
S(\theta) \geq S^{m}(\theta) \geq 0
$$

We assume $S^{m \prime}(\cdot)>0, S^{m}(0)=0$.

One simple setup which generates the payoff functions $S(\cdot), S^{m}(\cdot), \pi(\cdot)$ is the following. Suppose that the inverse demand function for the single good produced in the marketplace is given by $p=D(q, \theta)$, where $\theta$ is a shift parameter that affects the demand curve. Let $c$ denote the marginal cost of production. Here the social surplus is given by the area below the demand curve and above the cost curve:

$$
S(\theta)=\int_{c}^{\infty} D(x, \theta) d x
$$

The social surplus under monopoly is given by

$$
S(\theta)=\int_{p^{m}}^{\infty}\left[D(x, \theta)+\left(p^{m}-c\right)\right] d x
$$

where $p^{m}$ is the price chosen by a profit-maximizing monopolist. This simple example easily maps onto the general environment described above and generates the surplus function under the competitive markets $S(\theta)$, the surplus function under the monopoly $S^{m}(\theta)$, and the function for monopoly profits of the form $\pi(\theta)$.

\section{Benchmark with full information}

In this section, we set up a benchmark example of the environment in which the quality of an idea is known to the planner.

The classic analysis of the optimal patent length problem is the work of Nordhaus (1969). The planner seeks to maximize the discounted value of the social surplus. The only instrument available to the planner is a patent if length $\hat{T}$. The problem of the planner is to determine the length of time $\hat{T}$ that a patent will be valid, which solves the following problem:

$$
\max _{\hat{T}} \int\left\{\int_{0}^{\hat{T}} e^{-r t} S^{m}(\theta) d t+\int_{\hat{T}}^{\infty} e^{-r t} S(\theta) d t\right\} d F(\theta)
$$


s.t.

$$
\int_{0}^{\hat{T}} e^{-r t} \pi(\theta) d t \geq K
$$

In the objective function, the social surplus is equal to $S^{m}(\theta)$ for the time period between 0 and $\hat{T}$ as the good is produced by the monopoly under the patent granted. Afterward, the social surplus is equal to $S(\theta)$ as the good is produced under the competitive markets. The equation (1) is a participation constraint that guarantees that the innovator granted a patent of length $\hat{T}$ at least breaks even.

Letting $\tau=\int_{0}^{\hat{T}} e^{-r t} \pi(\theta) d t$, this problem reduces to

$$
\max _{\tau} \int\left[\tau S^{m}(\theta)+(1-\tau) S(\theta)\right] d F(\theta)
$$

s.t.

$$
\tau \pi(\theta) \geq K
$$

Suppose now that prizes are available, and prizes can be a function of the quality of the good. Then the problem of the social planner becomes that of maximizing (2) subject to

$$
\tau \pi(\theta)+T(\theta) \geq K
$$

where $T(\theta)$ represents the prize. Since a prize is a lump sum transfer financed by lump sum taxes on consumers, it does not affect the social surplus. The solution of the problem with prizes is then to set the patent length $\tau=0$ and reward innovators with prizes above the critical threshold value where the voluntary participation constraint binds. Thus, if the planner has as much information as the innovator, patents are never optimal. This reasoning leads us to consider the environments in which the planner has less information than private agents.

\section{Benchmark with private information}

Consider a benchmark model in which the quality of the idea $\theta$ is private information to the innovator. No other agents can observe $\theta$. The planner only observes whether the good has been produced or not. Since the innovator can always incur no cost and produce a good of type 0, the instruments available to the planner are, without loss of generality, the length of the patent $\tau$ and the lump-sum prize or transfer $T$.

We now define a mechanism design problem of the social planner as follows. From the 
revelation principle we can restrict attention to direct mechanisms which consist of a reported type $\theta \in[0, \bar{\theta}]$ for the innovator to the planner and the outcome functions $\delta(\theta), \tau(\theta), T(\theta)$. The function $\delta(\theta):[0, \bar{\theta}] \rightarrow\{0,1\}$ is an instruction from the planner to the innovator recommending whether or not to incur the fixed cost $K$. The patent length function is given by $\tau(\theta):[0, \bar{\theta}] \rightarrow[0,1]$. The prize function is given by $T(\theta):[0, \bar{\theta}] \rightarrow(-\infty, \infty)$.

These outcome functions induce the following payoffs for the innovator. Let $V(\theta, \hat{\theta}, \gamma)$ denote the profits of the innovator who has an idea of quality $\theta$, reports an idea of quality $\hat{\theta}$ to the planner, where $\gamma=1$ denotes that type $\theta>0$ good is produced, and $\gamma=0$ denotes that $\theta=0$ good is produced. The innovator's payoffs are given by

$$
V(\theta, \hat{\theta}, \gamma)=\delta(\hat{\theta})[\tau(\hat{\theta}) \pi(\gamma \theta)-\gamma K+T(\hat{\theta})]
$$

The social surplus for the planner under truth telling is given by

$$
W=\int\left\{\delta(\theta)\left[\tau(\theta) S^{m}(\theta)+(1-\tau(\theta)) S(\theta)-K\right]\right\} d F(\theta)
$$

The above equation states that for the period of length $\tau(\theta)$, the good is produced under monopoly so that the planner receives the surplus of $S^{m}(\theta)$, for the period of $(1-\tau(\theta))$ the good is produced by the competitive markets and the surplus of $S(\theta)$ is received.

A mechanism is incentive compatible if for all $(\theta, \hat{\theta})$ it satisfies

$$
V(\theta, \theta, \delta(\theta)) \geq \max _{\hat{\theta} \in[0, \bar{\theta}]} V(\theta, \hat{\theta}, \delta(\hat{\theta}))
$$

In this formulation of the incentive compatibility constraint, note that we require that an innovator who follows the recommendation of the planner, $\delta(\theta)$, and reports his true type gets a higher payoff than an innovator who deviates from the recommendation of the planner and chooses $\gamma \neq \delta(\theta)$ or misreports the type $\hat{\theta}$, or does both.

A mechanism satisfies voluntary participation if

$$
V(\theta, \theta, \delta(\theta)) \geq 0
$$


We say that the mechanism satisfies a no money pump assumption if

$$
T(0)=0
$$

This assumption is motivated by the following considerations. The economy has a large number of innovators with ideas of value $\theta=0$. If the mechanism gave positive prizes $T$ to all innovators, the society will then not be able to pay off for all of these ideas of no value.

We now formally define an interim-efficient mechanism.

Definition 1. The mechanism is interim efficient if it maximizes social surplus (3) subject to incentive compatibility (4), no money pump assumption (6), and voluntary participation (5).

We then have the following proposition.

Proposition 1. (Optimality of uniform patents). The interim-efficient mechanism has no prizes $T(\theta)=0, \forall \theta$, and a constant patent length $\tau(\theta)=\bar{\tau}, \forall \theta$.

Proof. We first show that there is some critical threshold $\theta^{*}$ such that $\delta(\theta)=0$ for $\theta<\theta^{*}$, and $\delta(\theta)=1$ for $\theta \geq \theta^{*}$. The argument is by contradiction. Suppose that $\theta_{1}<\theta_{2}, \delta\left(\theta_{1}\right)=1$, and $\delta\left(\theta_{2}\right)=0$. Consider the incentive compatibility constraint for the innovator who has an idea of quality $\theta_{2}$ and contemplates a deviation to reporting $\theta_{1}$. Under the supposition that $\delta\left(\theta_{2}\right)=0$, the payoff of the innovator of truth telling is equal to 0 . Using the incentive compatibility constraint, we then have the following sequence of inequalities leading to a contradiction:

$$
0 \geq \tau\left(\theta_{1}\right) \pi\left(\theta_{2}\right)+T\left(\theta_{1}\right)-K>\tau\left(\theta_{1}\right) \pi\left(\theta_{1}\right)+T\left(\theta_{1}\right)-K \geq 0 .
$$

Here, the first inequality is the incentive compatibility constraint. The second inequality follows because $\pi(\theta)$ is strictly increasing. The last inequality follows from the voluntary participation constraint of the type $\theta_{1}$. This argument establishes the critical threshold result.

Next we show that the incentive compatibility constraint implies that for the set of the innovated goods, the patent length is nondecreasing in the quality of the good $\theta$. Adding and substracting $\tau(\hat{\theta}) \pi(\hat{\theta})$ to the incentive compatibility constraint (4), we have that for any $\theta, \hat{\theta}$

$$
V(\theta, \theta, \delta(\theta)) \geq V(\hat{\theta}, \hat{\theta}, \delta(\hat{\theta}))+\tau(\hat{\theta})(\pi(\theta)-\pi(\hat{\theta}))
$$


A similar argument implies that:

$$
V(\hat{\theta}, \hat{\theta}, \delta(\hat{\theta})) \geq V(\theta, \theta, \delta(\theta))+\tau(\theta)(\pi(\hat{\theta})-\pi(\theta))
$$

These two inequalities imply that if $\theta>\hat{\theta}$, then $\tau(\theta) \geq \tau(\hat{\theta})$.

Then, since social surplus is decreasing in the length of the patent, having a constant patent length is optimal. Voluntary participation by the threshold type implies that

$$
\bar{\tau} \pi\left(\theta^{*}\right)+T\left(\theta^{*}\right)-K \geq 0
$$

Welfare maximization implies that the threshold type must satisfy

$$
\bar{\tau} S^{m}\left(\theta^{*}\right)+(1-\tau) S\left(\theta^{*}\right)-K \geq 0
$$

Because $S\left(\theta^{*}\right) \geq S^{m}\left(\theta^{*}\right) \geq \pi^{m}\left(\theta^{*}\right)$, it follows that welfare maximization implies that the voluntary participation of the threshold type $\theta^{*}$ is binding and the prize for the threshold type $T\left(\theta^{*}\right)=0$. Since the patent length is constant and $\pi(\theta)$ is increasing, then the incentive compatibility constraint implies that $T(\theta)=0, \forall \theta$. Q.E.D.

Notice that the interim-efficient mechanism yields the same allocations as the mechanism in Nordhaus (1969) described above. Here, however, we allow for the possibility of prizes in addition to patents. Incentive compatibility and welfare maximization imply that it is optimal not to use prizes or taxes but to use patents only. Hence, the result that only patents are used does not follow by assumption but rather by the need to provide incentives for innovation.

We now formally define a full information efficient mechanism.

Definition 2. A mechanism is ex post efficient (or full information efficient) if it maximizes the social surplus (3) subject to no money pump assumption (6) and the voluntary participation constraint (5).

It is immediate that the ex post efficient mechanism has no deadweight loss. Specifically, the ex post efficient mechanism has the planner recommending the innovator to innovate if $S(\theta) \geq K$. Note that the ex post efficient mechanism can be implemented by a variety of prizes. Specifically, any prize that satisfies $K \leq T(\theta)$ if $\delta(\theta)=1$ implements the ex post efficient outcomes. 


\section{Market signals, prizes, and patents}

Consider a version of the economy in which private agents other than the innovator receive signals about the quality of the good innovated. One can imagine a variety of schemes that elicit the information that other agents - or more generally, the markets — possess. Two specific schemes gained significant recent attention both theoretically and in policymaking circles.

Specifically, suppose that in addition to the innovator, another private agent, called a competitor, observes the value of the innovated good $\theta$ after it was innovated. In this environment, the planner can allow the length of the patent, and the prize/transfers depend on information revealed by the competitor about the quality of the good. Note that the decision on whether to incur the cost of innovation $K$ cannot depend on the information available to the competitor.

\section{A. Market signals without manipulation}

Let $\theta$ denote the report made by the innovator and $\delta(\theta)$ the recommendation by the mechanism to incur the fixed cost $K$. (Recall that if the cost is not incurred, the innovator produces a good of quality 0.) After the innovator produces the good, the competitor must submit a report of the quality of the good. Let $\theta^{c}$ denote the report made by the competitor. A mechanism consists of reports made by the innovator and the competitor and outcome functions $\delta(\theta), \tau\left(\theta, \theta^{c}\right), T\left(\theta, \theta^{c}\right)$, $T^{c}\left(\theta, \theta^{c}\right)$, where $\delta(\theta)$ denotes the recommendation by the mechanism to incur the cost $K ; \tau\left(\theta, \theta^{c}\right)$ denotes the length of the patent; $T\left(\theta, \theta^{c}\right)$ denotes the prize to the innovator; and $T^{c}\left(\theta, \theta^{c}\right)$ denotes transfer to the competitor.

The payoffs to the innovator induced by the mechanism are then given by

$$
V\left(\theta, \hat{\theta}, \theta^{c}(\gamma), \gamma\right)=\delta(\hat{\theta})\left[\tau\left(\hat{\theta}, \theta^{c}(\gamma)\right) \pi(\gamma \theta)-\gamma K+T\left(\hat{\theta}, \theta^{c}\right)\right]
$$

In this formulation of the payoff to the innovator, $V\left(\theta, \hat{\theta}, \theta^{c}(\gamma), \gamma\right)$, the arguments are, in order, the true type of the quality of the good $\theta$, the report by the innovator $\hat{\theta}$, the report by the competitor $\theta^{c}(\gamma)$, and the decision of the innovator $\gamma$ to incur the cost $K$. Note that the report of the competitor $\theta^{c}(\gamma)$ depends on whether or not the innovator incurs the cost $K$. The payoffs to the competitor are given by $T^{c}\left(\theta, \theta^{c}\right)$.

The incentive compatibility constraint for the innovator is given by

$$
V(\theta, \theta, \theta, \delta(\theta)) \geq \max _{\hat{\theta} \in[0, \bar{\theta}]} V(\theta, \hat{\theta}, \theta, \delta(\hat{\theta}))
$$


Note that in this incentive compatibility constraint we have assumed that the competitor reveals the information truthfully. This formulation of the incentive compatibility constraint follows from the revelation principle that states that the Bayesian equilibrium of any game can be implemented as a truth-telling equilibrium of a direct mechanism.

The incentive compatibility constraint for the competitor is given by

$$
T^{c}(\theta, \theta) \geq T^{c}\left(\theta, \hat{\theta}^{c}\right)
$$

An interim-efficient and the ex post efficient mechanisms are defined in the analogous way as in the above. Note that since ex post efficiency assumes that the planner has the same information as the private agents, the ex post efficient outcomes in the environment with and without market signals are identical.

In the following proposition, we show that the patents are never optimal. In fact, the full information optimum can be achieved solely with prizes.

Proposition 2. (Optimality of prizes) In the environment with market signals, the interimefficient mechanism is ex post efficient.

Proof. Let the planner's recommendation be to produce the good when the social value is higher than fixed costs: $\delta(\theta)=1$ if $S(\theta) \geq K ; \delta(\theta)=0$ otherwise. Let $T_{1}(\theta, \theta) \geq K$ if $\delta(\theta)=1$; $T_{1}\left(\theta, \theta^{c}\right)=0$ if $\theta \neq \theta^{c}$. Let $T_{2}\left(\theta, \theta^{c}\right)=0$. In other words, implement the full information outcomes associated with the value of $\theta$ only if both agents report that same value of $\theta$. If the agents disagree, then give the innovator a transfer equal to zero. The competitor always receives the same transfer regardless of his report. Then the best response of the competitor is to report the value of the innovated goods truthfully. Q.E.D.

Note that above we restricted the planner to award the patent only to the innovator. A more general setup would allow the planner to reward the competitor with the patent. This restriction is without loss of generality, since proposition (2.) shows that the planner can achieve the full information outcome.

So far, we have assumed that the competitor receives the same signal as the innovator. Suppose now that the competitor receives a noisy, but unbiased, signal $s$ of the quality of the good so that $E(\theta \mid s)=s$ and that $E(s \mid \theta)=\theta$. Consider a mechanism which sets the prize to the innovator $T_{1}(\theta, s)=s$ if $S(s) \geq K$ and 0 otherwise and sets the transfer to the competitor to 0 . 
Since the innovator is risk-neutral, this mechanism yields the ex post efficient level of innovation as a truth-telling outcome.

The competitor's report also has an immediate market interpretation. Consider the simple market setup described above in which the inverse demand for the good is given by $p=D(q, \theta)$ and $c$ is the marginal cost of production. Suppose the market consists of a large number of producers, all of whom can produce the good at marginal cost. The mechanism designer then makes the knowledge of how to produce the good freely available to all producers and asks each producer to report sales of the good. Since the price $p$ equals the marginal cost of production $c$ in a competitive market, aggregate sales $q$ can then be used to uncover the market size parameter $\theta$.

Note that we have also assumed that the cost of innovating is known to the designer. Our results extend readily to the cse in which this cost is drawn from some distribution, say, $G(K)$ and is private information to the innovator. To see this extension, consider a mechanism in which the innovator's prize is given by the social surplus if the innovator's and competitor's reports agree, so that $T_{1}(\theta, \theta)=S(\theta)$ and the innovator receives no prize if the reports disagree so that $T_{1}\left(\theta, \theta^{c}\right)=0$ if $\theta \neq \theta^{c}$. Clearly, truth telling is incentive compatible and the mechanism implements the efficient allocation in the sense that $\delta(\theta)=1$ if and only if $S(\theta) \geq K$.

\section{B. Unique implementation of prize mechanisms}

The mechanism that we have discussed uses information from the competitor to reward the innovator. Under our particular mechanism, the competitor is indifferent about what information to report. Truth telling is one of the equilibria of the game. Typically, the game has many other equilibria. A natural question is whether we can design a mechanism which is ex post efficient and has a unique equilibrium. Here, we adapt the mechanism of Moore and Repullo (1988) to our environment. We show that such a mechanism has a unique subgame-perfect equilibrium in which both the innovator and the competitor report the truth.

The mechanism has two stages. In Stage 1, the innovator and the competitor make reports

to the planner. Denote the report of the innovator by $\theta_{1}$ and that of the competitor by $\theta^{c}$. If $\theta_{1}=\theta^{c}$, equals say $\theta$, then implement the ex post efficient outcome associated with the common report $\theta$. If $\theta_{1} \neq \theta^{c}$, then move to Stage 2. In Stage 2, the innovator is given a choice between two alternatives, denoted by A and B. In each alternative, the innovator is granted a patent with the 
length $\mu_{A}\left(\theta_{1}, \theta^{c}\right)$ and $\mu_{B}\left(\theta_{1}, \theta^{c}\right)$ and prizes $T_{A}\left(\theta_{1}, \theta^{c}\right)$ and $T_{B}\left(\theta_{1}, \theta^{c}\right)$ chosen to satisfy

$$
\begin{array}{r}
\max \left\{\mu_{A}\left(\theta_{1}, \theta^{c}\right) \pi\left(\theta_{1}\right)-K+T_{A}\left(\theta_{1}, \theta^{c}\right) ; T_{A}\left(\theta_{1}, \theta^{c}\right)\right\} \\
>\max \left\{\mu_{B}\left(\theta_{1}, \theta^{c}\right) \pi\left(\theta_{1}\right)-K+T_{B}\left(\theta_{1}, \theta^{c}\right) ; T_{B}\left(\theta_{1}, \theta^{c}\right)\right\},
\end{array}
$$

and

$$
\begin{gathered}
\max \left\{\mu_{B}\left(\theta_{1}, \theta^{c}\right) \pi\left(\theta^{c}\right)-K+T_{B}\left(\theta_{1}, \theta^{c}\right) ; T_{B}\left(\theta_{1}, \theta^{c}\right)\right\} \\
>\max \left\{\mu_{A}\left(\theta_{1}, \theta^{c}\right) \pi\left(\theta_{1}\right)-K+T_{A}\left(\theta_{1}, \theta^{c}\right) ; T_{A}\left(\theta_{1}, \theta^{c}\right)\right\}, \\
T\left(\theta^{c}\right)-K>\max \left\{\mu_{B}\left(\theta_{1}, \theta^{c}\right) \pi\left(\theta^{c}\right)-K+T_{B}\left(\theta_{1}, \theta^{c}\right) ; T_{B}\left(\theta_{1}, \theta^{c}\right)\right\} .
\end{gathered}
$$

The basic idea behind this mechanism is that in the second stage, the innovator is given an option to rescind on his previous report at a cost. The first inequality ensures that if $\theta_{1}$ is the true report and $\theta^{c}$ is not, the innovator will choose alternative $A$. The second inequality ensures that if $\theta^{c}$ is the true report and $\theta_{1}$ is not, the innovator will choose alternative $B$. The third inequality ensures that if the competitor tells the truth, the innovator also tells the truth and finds it optimal not to go to Stage 2. Since four choice variables need to satisfy only three inequalities, clearly we can choose these four variables.

Now we turn to the transfers to the competitor. If both agents report the same value of $\theta$ in Stage 1, the competitor receives a transfer of zero. If the reports differ, then the competitor pays a $\operatorname{tax}-\underline{T}$ if the innovator chooses an alternative $A$ and receives a transfer $\bar{T}$ if the innovator chooses an alternative $B$.

We claim that this mechanism has a unique equilibrium that is truth telling. Suppose that the equilibrium for some realized value of $\theta$ involves these two agents reporting a common value of $\hat{\theta} \neq \theta$. Under this supposed equilibrium, the payoff of the competitor is equal to zero. Now consider a deviation by the competitor to the true report, that is setting $\theta^{c}=\theta$. Under this deviation, the mechanism requires the players to proceed to Stage 2. Inequality (10) guarantees that in this subgame, the innovator will optimally choose the alternative $B$. Recall that if the innovator chooses the alternative $B$, the competitor receives a positive transfer. Thus, such deviation is profitable and the equilibrium cannot have both agents reporting a common value $\hat{\theta} \neq \theta$.

Now suppose that the innovator reports the truth and the competitor lies and reports a value of $\hat{\theta} \neq \theta$. The mechanism requires that the players move to Stage 2. In that stage, inequality (9) 
guarantees that in Stage 2, the innovator will choose option $A$. The competitor's payoff is then given by the tax that the competitor must pay. A deviation of the competitor to reporting the truth gives the competitor a zero payoff which dominates misreporting. Thus, we cannot have an equilibrium in which the innovator tells the truth and the competitor lies.

Next suppose that the competitor reports the truth and the innovator lies and reports a value of $\hat{\theta} \neq \theta$. The mechanism requires that the players move to Stage 2. In that stage, inequality (10) guarantees that the innovator will choose option $B$. The innovator's payoff is then given by the left-hand side of (10) equal to the right-hand side of (11). Consider a deviation from the supposed equilibrium in which the innovator reports the truth. The payoff to this deviation is given by the left-hand side of (11). Thus, this deviation is profitable and the game cannot have an equilibrium in which the competitor reports the truth and the innovator lies.

This argument establishes the following proposition on a unique implementation of the ex post efficient equilibrium.

Proposition 3. Consider the game in which the innovator and the competitor both receive the same signal about the quality of the good to be innovated. There exists a mechanism which has truth telling by both agents and which implements the ex post efficient outcome.

\section{Market signals with bribes}

We now consider an environment in which the innovator can bribe the competitor to misreport the quality of the good. We show that in this environment, the equilibrium outcomes coincide exactly with those in the environment in which no agent other than the innovator observes the quality of the good. This result implies that patents are again optimal as in Proposition 1..

We begin by describing how the possibility of bribes modifies the constraints that the social planner faces. We do so by considering an arbitrary mechanism which consists of action sets $A$ for the innovator and $A^{C}$ for the competitor, actions $a \in A$ and $a^{c} \in A^{c}$, recommendations by the planner to innovate $\delta\left(a_{1}\right)$, length of patent granted to the innovator $\tau\left(a, a^{c}\right)$, length of the patent awarded to the competitor $\tau^{c}\left(a, a^{c}\right)$, and the prizes $T\left(a, a^{c}\right)$ and $T^{c}\left(a, a^{c}\right)$.

We assume that the players can observe each other's actions. We also assume that they can agree, before the actions are chosen, to pay transfers (bribes) to each other contingent on the actions chosen by the innovator and the competitor. We assume that these bribes are not observable to the mechanism designer and that there are no limits to the size of the bribes. Let $B\left(a, a^{c}, \theta\right)$ and 
$B^{c}\left(a, a^{c}, \theta\right)$ denote the payments made by the innovator and the competitor so that

$$
B\left(a, a^{c}, \theta\right)+B^{c}\left(a, a^{c}, \theta\right)=0 .
$$

Note that we assume that these bribes can be enforced. The payoffs of the agents are augmented with the bribes. The revelation principle clearly holds in this environment so that any Nash equilibrium of the arbitrary mechanism can be implemented by a direct mechanism. Let $V\left(\theta, \hat{\theta}, \hat{\theta}^{c}, \delta(\hat{\theta})\right)$ and $V^{c}\left(\theta, \hat{\theta}, \hat{\theta}^{c}, \delta(\hat{\theta})\right)$ denote the payoffs granted by the direct mechanism to the innovator and the competitor. These payoffs are given by

$$
V\left(\theta, \hat{\theta}, \hat{\theta}^{c}(\gamma), \gamma\right)=\gamma\left[\tau\left(\hat{\theta}, \hat{\theta}^{c}(\gamma)\right) \pi(\gamma \theta)-\gamma K+T\left(\hat{\theta}, \theta^{c}\right)\right]
$$

and the payoffs to the competitor are given by

$$
V^{c}\left(\theta, \hat{\theta}, \hat{\theta}^{c}(\gamma), \gamma\right)=\gamma\left[\tau_{c}\left(\hat{\theta}, \hat{\theta}^{c}(\gamma)\right) \pi(\gamma \theta)+T^{c}\left(\hat{\theta}, \theta^{c}\right)\right]
$$

Note that these payoffs do not include the bribes. When augmented by the bribes, the payoffs are given by $V\left(\theta, \hat{\theta}, \hat{\theta}^{c}(\gamma), \gamma\right)+B\left(\theta, \hat{\theta}, \hat{\theta}^{c}(\gamma), \gamma\right)$ to the innovator and $V^{c}\left(\theta, \hat{\theta}, \hat{\theta}^{c}(\gamma), \gamma\right)+B^{c}\left(\theta, \hat{\theta}, \hat{\theta}^{c}(\gamma), \gamma\right)$. Here, $\theta$ denotes the quality of the idea, $\hat{\theta}$ denotes the report by the innovator, and $\hat{\theta}^{c}$ denotes the report by the competitor. We now prove the lemma as follows.

Lemma 1. The truth-telling equilibrium of any direct mechanism must satisfy the bribe-proofness condition:

$$
V(\theta, \theta, \theta, \delta(\theta))+V^{c}(\theta, \theta, \theta, \delta(\theta)) \geq V\left(\theta, \hat{\theta}, \hat{\theta}^{c}, \delta(\hat{\theta})\right)+V^{c}\left(\theta, \hat{\theta}, \hat{\theta}^{c}, \delta(\hat{\theta})\right)
$$

Proof. The proof is by contradiction. Suppose for some $\theta, \hat{\theta}$, and $\hat{\theta}^{c}$, truth telling is an equilibrium and the bribe-proofness condition (15) is not satisfied. Suppose that, at the report $\hat{\theta}, \hat{\theta}^{c}$, the innovator is strictly better off if both misreport so that $V\left(\theta, \hat{\theta}, \hat{\theta}^{c}, \delta(\hat{\theta})\right)>V(\theta, \theta, \theta, \delta(\theta))$ and the competitor is strictly worse off so that $V^{c}\left(\theta, \hat{\theta}, \hat{\theta}^{c}, \delta(\hat{\theta})\right)<V^{c}(\theta, \theta, \theta, \delta(\theta))$. Consider a bribe by the innovator that offers the competitor all the surplus the innovator gains by misreporting, so that the bribe equals $V\left(\theta, \hat{\theta}, \hat{\theta}^{c}, \delta(\hat{\theta})\right)-V(\theta, \theta, \theta, \delta(\theta))$. Since, by assumption, (15) does not hold, this bribe makes the competitor's payoffs higher than under truth telling, so that truth telling is not an equilibrium. We have a contradiction. Q.E.D. 
Note that this lemma relies upon the assumption that the bribe payments are not observable to the mechanism designer. Note also that the proof of this lemma fails if the size of bribes is limited. To see that the lemma does not hold if bribes are limited, note that if we restrict bribes to be zero, this environment reduces to the one without market manipulation. In that environment, the efficient outcome violates (15).

We use this lemma to show that the solution to the social planner's problem in this environment with bribes coincides with the solution to that in the environment without market signals.

The incentive compatibility constraint for the innovator is given by

$$
V(\theta, \theta, \theta, \delta(\theta)) \geq \max _{\hat{\theta} \in[0, \bar{\theta}]} V(\theta, \hat{\theta}, \theta, \delta(\hat{\theta}))
$$

and the incentive compatibility constraint for the competitor is given by

$$
V^{c}(\theta, \theta, \theta, \delta(\theta)) \geq \max _{\hat{\theta} \in[0, \bar{\theta}]} V^{c}\left(\theta, \theta, \hat{\theta}^{c}, \delta(\theta)\right)
$$

We denote the sum of the payoffs to the innovator and the competitor by the social planner:

$$
\bar{V}(\theta)=V(\theta, \theta, \theta, \delta(\theta))+V^{c}(\theta, \theta, \theta, \delta(\theta))
$$

The bribe-proofness constraint is now given by

$$
\bar{V}(\theta) \geq \max _{\hat{\theta}, \hat{\theta}^{c}}\left[V\left(\theta, \hat{\theta}, \hat{\theta}^{c}, \delta(\hat{\theta})\right)+V^{c}\left(\theta, \hat{\theta}, \hat{\theta}^{c}, \delta(\hat{\theta})\right)\right]
$$

The social planner's payoffs in the truth-telling equilibrium are now given by

$$
W=\int\left\{\delta(\theta)\left[\bar{\tau}(\theta) S^{m}(\theta)+(1-\bar{\tau}(\theta)) S(\theta)-K\right]\right\} d F(\theta)
$$

where

$$
\bar{\tau}(\theta)=\tau(\theta)+\tau^{c}(\theta) .
$$

The social planner's problem is to maximize (18) subject to (16), (17), (15), and the analogs of the voluntary participation and no money pump constraints. We now show the proposition that characterizes the social planner's problem.

Proposition 4. (Optimality of patents with bribes) The solution to the social planner's prob- 
lem with bribes coincides with that in the environment with no market signals problem. In particular, the solution to the social planner's problem necessarily has $\tau(\theta)>0$ if $\delta(\theta)=1$.

Proof. Consider a relaxed version of the social planner's problem which does not impose the individual incentive compatibility constraints and which replaces the voluntary participation and the no money pump assumption by two constraints - one that requires that the sum of the payoffs to the innovator and the competitor is non-negative, and the other that the sum of transfers to them is non-positive. This formulation of the problem is identical to the social planner's problem in the environment with no market signals. To see that the formulations are identical, note that, using (13) and (14), (15) can now be written as

$$
\bar{V}(\theta) \geq \max _{\hat{\theta}, \hat{\theta}^{c}}\left[\gamma\left[\left(\tau\left(\hat{\theta}, \hat{\theta}^{c}(\gamma)\right)+\tau^{c}\left(\hat{\theta}, \hat{\theta}^{c}(\gamma)\right)\right) \pi(\gamma \theta)-\gamma K+T\left(\hat{\theta}, \theta^{c}\right)+T^{c}\left(\hat{\theta}, \theta^{c}\right)\right]\right]
$$

This incentive constraint is clearly effectively the same as (8). Thus, any solution to the social planner's problem in the environment with bribes can yield surplus no greater than in the relaxed problem. Consider the following mechanism which is clearly feasible in the social planner's problem. In this mechanism, the competitor's reports are ignored and the competitor receives zero transfers. Thus, the solutions to the two problems coincide. The last part of the proposition follows immediately from Proposition (1.). Q.E.D.

\section{A. Implicit collusion as a form of bribes}

One interpretation of bribes is that they are implicit payments sustained by a form of implicit collusion. An example of such implicit collusion is as follows. Suppose that the economy has two agents and lasts for an infinite number of periods. Agents discount the future at the rate $\beta$. With probability 0.5 , one of these agents is the innovator and the other is the competitor in each period. Suppose that the planner chooses some mechanism. Fix an equilibrium of this infinitely repeated mechanism. The bribe paid by the innovator to the competitor can now be thought as the difference between the payoffs in this equilibrium and the best equilibrium. Suppose that the payoffs in any equilibrium are bounded above and that the differences in the payoffs in the best and the worst equilibria are given by $\bar{B}$. We argue that the planner can always choose a mechanism which induces truth telling. Specifically, suppose that the planner chooses in each period a mechanism similar to the Moore and Repullo (1998) mechanism described above. Let the planner set the Stage 2 transfers

to the competitor at $\bar{T}>\bar{B}$. By the same argument as in the section on the unique implementation 
in the single period game, it follows that the competitor always tells the truth regardless of the innovator's strategies. Thus, manipulation in the form of implicit collusion alone does not suggest that patents are optimal.

\section{Limitations of Kremer's mechanism when manipulation is possible}

Kremer (1998) describes a mechanism intended to exploit information that other market participants may have regarding the value of the innovation. Kremer's mechanism is as follows. A patent holder has an option of choosing to be a part of the auction mechanism. The government chooses a second price auction. The winner of the second price auction receives a patent with probability $\epsilon$ and in that event pays the second-highest bid. With probability $(1-\epsilon)$, the patent is invalid, and the information associated with the patent is placed in the public domain. In either event, the innovator receives a prize which is a multiple (a markup) of the second-highest bid.

Suppose that the value of $\theta$ is observed by the innovator and all market participants. Without market manipulation, Kremer's mechanism leads to better outcomes than under the patent system with probability $(1-\epsilon)$ and to no worse outcomes with probability $\epsilon$, if the prize multiple is sufficiently high. The usual argument for this result is that in the second price auction, the dominant strategy equilibrium is for all bidders to bid the true value of the object. Thus, all bidders bid $\mu \pi^{m}(\theta)$, where $\mu$ is the length of the patent and $\pi^{m}(\theta)$ denotes monopoly profits. As long as the prize markup is greater than 1 , the patent holder finds it optimal to accept the auction mechanism. Indeed, this mechanism can lead some innovators to choose to innovate under the auction mechanism when they have not chosen under the patent system. It is, however, possible to set the markup so as to induce the efficient level of innovation. Note that for this mechanism to yield good outcomes, it is important that $\epsilon>0$, because if $\epsilon=0$, the market participants have no incentive to submit any bids. Equilibria in which the participants submit no bids are possible and likely to occur, especially if bidding is costly.

Next consider a possibility of market manipulation. Formally, we assume that the innovator can participate directly in Kremer's auction or can designate an agent whom we call an accomplice to bid on his behalf. Now suppose that each other market participant $i$ receives a signal $s_{i}$ from a distribution $G(s \mid \theta)$. These signals are independent across market participants conditional on the innovator's type $\theta$. Without loss of generality, suppose that $E\left(s_{i} \mid \theta\right)=\theta$.

We will show that in any equilibrium of this game, the innovator (or his accomplice) wins the auction and that the second-highest bid is zero. To see that this outcome is an equilibrium, 
suppose that one of the market participants wins the auction. In order for this outcome to be an equilibrium, the market participant must submit a bid higher than the innovator's bid. If the market participant's bid is higher than the true value of the innovation, then that market participant is better off bidding zero. If the other participant's bid is below the patent's value to the innovator, the innovator should raise his bid to the true value. Furthermore, note that if $\epsilon$ is strictly positive, conditional on innovating, the innovator should participate in the auction so as to retain the patent in the event that the auction mechanism is not used to give the patent away. That is, the innovator cannot credibly commit not to participate in the auction. Realizing that this will be the equilibrium outcome if the innovator submits the patent for the auction, the equilibrium strategy for the innovator is not to submit the patent to the auction mechanism. Then, the auction mechanism will not help in reducing deadweight losses due to the patent system. Other forms of manipulation lead to different equilibrium outcomes but share the property that the auction mechanism will not help solve the problem of reducing the deadweight losses. To see the effects of other forms of manipulation, suppose that the innovator can designate two accomplices to the auction mechanism. Then the equilibrium outcome in the auction mechanism is for each accomplice to bid the maximal amount permitted by the auction mechanism. The other market participants then bid zero. These strategies are clearly best responses. Yet, the mechanism leads to too much innovation compared to the optimum.

\section{Market signals with costly manipulation}

We have shown that if the innovator can manipulate market signals by bribing other participants, patents are optimal. We now examine economies in which the innovator can manipulate the market signals in other ways. We show that if manipulation is costless, then patents are optimal. If manipulation is very costly, prizes are optimal. For intermediate ranges, a combination of prizes and patents is optimal.

We begin by describing a fairly abstract environment in which the planner receives the signal $s$ about the quality of the good innovated. The innovator can manipulate the signal by incurring the cost. Specifically, if the planner receives the signal $s$, the cost to the innovator of manipulating the signal is given by $c(s-\theta)$. Note that if the innovator does not manipulate the signal, then $s=\theta$, so the signal reveals the quality of the good perfectly. With this formulation, the payoffs of 
an innovator who has an idea of quality $\theta$ and chooses to report the idea of quality $\hat{\theta}$ are given by

$$
V^{m}(\theta, \hat{\theta}, \gamma)=\delta(\hat{\theta})[\tau(\hat{\theta}) \pi(\gamma \theta)-\gamma K+T(\hat{\theta})-c(\theta-\hat{\theta})]
$$

Incentive compatibility now becomes

$$
V^{m}(\theta, \theta, \delta(\theta)) \geq \max _{\hat{\theta} \in[0, \bar{\theta}]} V^{m}(\theta, \hat{\theta}, \delta(\hat{\theta}))
$$

The social planner's payoff, voluntary participation, and the no money pump constraints are unchanged. The social planner now maximizes the social surplus subject to the incentive compatibility constraint (20), voluntary participation (5), and the no money pump constraints (6).

Let $S\left(\theta^{*}\right)=K$ denote the threshold value of the quality of the good such that if $\theta \geq \theta^{*}$, the full information efficient mechanism requires that the good be innovated, $\delta(\theta)=1$. If $\theta \leq \theta^{*}$, then the good is not innovated, $\delta(\theta)=0$. We then have the following proposition. We show that if the manipulation costs are sufficiently high, patents are not optimal. If the manipulation costs are sufficiently low, the patents are used in any efficient mechanism.

Proposition 5. If $c \geq \frac{K}{\theta^{*}}$, then the solution to the social planner's problem can be implemented with prizes alone. If $c<\frac{K}{\theta^{*}}$, then the solution to the social planner's problem necessarily requires using patents.

Proof. First, suppose that $c \geq \frac{K}{\theta^{*}}$. Consider the following mechanism that sets $T(\theta)=K$ if $\theta \geq \theta^{*}$; $T(\theta)=0$, otherwise; $\delta(\theta)=1$ if and only if $\theta \geq \theta^{*}$. We will show that this mechanism is incentive compatible. Consider a reporting problem of an innovator with the quality of idea $\theta<\theta^{*}$. Truth telling yields a payoff of zero for this innovator. Suppose that this innovator deviates, claims that the quality of his idea is $\hat{\theta} \geq \theta^{*}$ and produces a good of quality $\hat{\theta}$. The payoff from such deviation is given by

$$
V^{m}(\theta, \hat{\theta}, 1)=-K+K-c(\hat{\theta}-\theta)=-c(\hat{\theta}-\theta)<0
$$

Thus, this deviation is not incentive compatible.

Suppose next that the innovator deviates and claims that the quality of the idea $\hat{\theta} \geq \theta^{*}$ and does not incur the cost $K$, thereby producing a good of quality 0 . The payoff from such a deviation is given by

$$
V^{m}(\theta, \hat{\theta}, 1)=K-c(\hat{\theta}-0) \leq K-c \theta^{*} \leq 0
$$


Thus, this deviation is not incentive compatible either.

Next suppose that $c<\frac{K}{\theta^{*}}$. The proof is by contradiction. Suppose that the welfaremaximizing mechanism does not require patents and specifies some prize function $T(\theta)$. Such a mechanism will clearly not have an innovation for $\theta<\theta^{*}$. Since a mechanism which only uses patents is feasible and has innovation for some values of $\theta$, the welfare-maximizing mechanism also has an innovation for some value of $\theta$. Suppose that for some value of $\theta \geq \theta^{*}$, the mechanism specifies $\delta(\theta)=1$ and some prize $T(\theta)$. Voluntary participation implies that

$$
T(\theta) \geq K
$$

Consider the incentive compatibility constraint for the innovator who has an idea of quality 0 and contemplates deviation to this value of $\theta$. Incentive compatibility requires

$$
0 \geq T(\theta)-c(\theta-0) \geq K-c \theta \geq K-c \theta^{*}
$$

Since $K-c \theta^{*}>0$, we have a contradiction. This mechanism is not incentive compatible. Q.E.D.

\section{A. A simple example}

We now consider a simple example which demonstrates that as the cost of manipulating the signal rises, the length of the patent falls. To do so, we suppose that the quality of the ideas takes three values: $0<\theta_{1}<\theta_{2}$. Suppose that the $S\left(\theta_{1}\right)<K$ and $S\left(\theta_{2}\right)>K, S\left(\theta_{2}\right)>S^{m}\left(\theta_{2}\right)>K$,

and that $c<\frac{K}{\theta_{2}}$. From Proposition 5. we know that the mechanism must feature patents. Since $S\left(\theta_{1}\right)<K$, it is optimal to have no innovation if the quality of the idea is $\theta_{1}$. Therefore, the incentive compatibility constraint that ensures that the innovator of quality $\theta_{1}$ does not misreport the quality of the idea and manipulate the signal is given by

$$
0 \geq \tau\left(\theta_{2}\right) \pi\left(\theta_{1}\right)-K+T\left(\theta_{2}\right)-c\left(\theta_{2}-\theta_{1}\right)
$$

The incentive compatibility constraint that the innovator of type $\theta=0$ does not misreport the quality of the idea and manipulate the signal is given by

$$
0 \geq T\left(\theta_{2}\right)-c\left(\theta_{2}-0\right)
$$


Note that this incentive compatibility constraint is also the incentive compatibility constraint for the innovator with the idea $\theta_{1}$ who chooses not to incur the cost and to misreport the signal.

The voluntary participation constraint for type $\theta_{2}$ is given by

$$
\tau\left(\theta_{2}\right) \pi\left(\theta_{2}\right)+T\left(\theta_{2}\right) \geq K
$$

The no money pump assumption and the voluntary participation constraints for type 0 and type $\theta_{1}$ imply that

$$
T(0)=T\left(\theta_{1}\right)=0
$$

The social surplus is given by

$$
\tau\left(\theta_{2}\right) S^{m}\left(\theta_{2}\right)+(1-\tau) S\left(\theta_{2}\right)-K
$$

Clearly, social surplus is maximized by making $\tau\left(\theta_{2}\right)$ as small as possible subject to the incentive compatibility and the voluntary participation constraints. Since reducing $\tau\left(\theta_{2}\right)$ relaxes (21), it follows that the voluntary participation constraint (23) must be binding so that

$$
\tau\left(\theta_{2}\right) \pi\left(\theta_{2}\right)+T\left(\theta_{2}\right)=K
$$

Substituting for $T\left(\theta_{2}\right)$ from (25) into (21), we have

$$
0 \geq \tau\left(\theta_{2}\right) \pi\left(\theta_{1}\right)-\tau\left(\theta_{2}\right) \pi\left(\theta_{2}\right)-c\left(\theta_{2}-\theta_{1}\right)
$$

Since the right side of this inequality is strictly negative, it follows that (21) is not binding at the optimum. Thus, (23) and (22) must be binding so that $T\left(\theta_{2}\right)=c \theta_{2}$. From these constraints we have

$$
\tau\left(\theta_{2}\right)=\frac{K-c \theta_{2}}{\pi\left(\theta_{2}\right)}
$$

We have shown that the length of the patent $\tau\left(\theta_{2}\right)$ is strictly decreasing in the manipulation $\operatorname{cost} c$. 


\section{Conclusion}

Our paper provides a comprehensive mechanism design treatment of providing incentives for innovation. We explored how information available to the competitors of the innovator and market information can be used by the mechanism designer to create such incentives. Our focus is also on the various ways in which information may be manipulated and on designing optimal mechanisms which are robust to such manipulation. 


\section{References}

Abramowicz, Michael. 2003. Perfecting patent prizes. Vanderbilt Law Review 56 (January): 115236.

Experts Group Report. 2008. Advance market commitments for pneumococcal vaccines. Presentation to the Donor Committee.

Guell, Robert C. and Marvin Fischbaum. 1995. Toward allocative efficiency in the prescription drug industry. Milbank Quarterly 73 (2): 213-230.

Hopenhayn, Hugo, Gerard Llobet, and Matthew Mitchell. 2006. Rewarding sequential innovators: Prizes, patents, and buyouts. Journal of Political Economy 114 (December): 1041-1068.

Kieff, F. Scott. 2001. Property rights and property rules for commercializing inventions. Minnesota Law Review 85: 697-754.

Kremer, Michael. 1998. Patent buyouts: A mechanism for encouraging innovation. Quarterly Journal of Economics 113 (November): 1137-1167.

Lichtman, Douglas G. 1997. "Pricing Prozac: Why the government should subsidize the purchase of patented pharmaceuticals. Harvard Journal of Law and Technology 11 (Fall): 123-139.

Moore, John, and Rafael Repullo. 1998. Subgame perfect implementation. Econometrica 56 (September): $1191-1220$

Nordhaus, William D. 1969. Invention, growth, and welfare: A theoretical treatment of technological change. Cambridge, MA: MIT Press.

Scotchmer, Suzanne. 2004. Innovation and incentives. Cambridge, MA: MIT Press.

Shavell, Steven, and Tanguy van Ypersele. 2001. Rewards versus intellectual property rights. Journal of Law and Econonomics 44 (October): 525-547.

Wright, Brian D. 1983. The economics of invention incentives: Patents, prizes, and research contracts. American Economic Review 73 (September): 691-707. 